\title{
Streamlining Differential Diagnosis of Coronary Heart Disease in a Pri- mary Care Clinic: Atypical and Typical Symptoms and Their Combina- tions
}

\author{
Gideon Charach*, Pavel Rabinovich and Moshe Weintraub
}

\author{
Department of Internal Medicine "C", Tel Aviv Sourasky Medical Center, Sackler Faculty of Medicine, Tel Aviv \\ University, Tel Aviv, Israel
}

\begin{abstract}
Background: We sought to identify the most predictive symptoms of coronary heart disease (CHD) in the setting of a very busy community primary care clinic. By examining the variability, distribution and frequency of symptoms singly and in combination we hoped to streamline the process of correctly identifying a patient presenting with CHD. Methods: All 449 patients suspected of having CHD by one primary physician (1995-2004) were evaluated by thallium scan, treadmill ergometry, and/or coronary angiography, as well as ECG and creatine phosphokinase /troponin levels. Group 1 included 216 subjects (117 males, 98 females, mean age 69.7 \pm 11.2 years) diagnosed as having acute coronary syndrome, stable angina pectoris or acute myocardial infarction. Group 2 included 233 (126 males, 108 females, mean age $65.2 \pm 12.1$ years) subjects whose comprehensive evaluations ruled out CHD. Results: The leading symptom in Groups 1 and 2 was chest pain [80\%] vs [80.3\%] (NS). Dyspnea was present in 10\% and 5\%; (p<0.037) patients, and jaw pain in $11 \%$ and $1 \%$ $(\mathrm{p}<0.0001)$ patients. The symptom combinations found more frequently in Group 1 were chest pain+jaw pain $(7 \%$ vs 1 . $04 \%$; $\mathrm{p}<0.0001)$ and dyspnea+sweating $(2.8 \%$ vs $0 \%, \mathrm{p}<0.01)$. Conclusion: Isolated symptoms (jaw pain, dyspnea) and combined symptoms (chest pain+jaw pain, dyspnea+sweating) were more specific for CHD than classical chest pain.
\end{abstract}

\section{INTRODUCTION}

The expression of symptoms related to CHD differs with age, gender and ethnicity [1-3]. For instance, Goldberg et al $[1,2]$ reported that there were fewer "classical" complaints among women than in men. Cardiac effort pain may occasionally be experienced predominantly in the neck or jaw [4]. Existing data on the significance and specificity of different symptoms of CHD at presentation are still inconclusive, and so clinicians must be familiar with the numerous characteristic as well as atypical symptoms of CHD for enhanced early diagnosis $[4,5]$. The increasing prevalence of angina pectoris and CHD among the growing numbers of elderly people together with the often life-threatening repercussions of mis diagnosis make it imperative to hone the diagnostic skills of the primary physician, especially in cases of atypical pain.

The aim of this study was to facilitate early correct identification of CHD by examining the variability, distribution and frequency of symptoms singly and in combination, in terms of cardiac risk factors. We wanted to identify the symptoms most predictive of CHD in the setting of a very busy community primary care clinic.

\section{METHODS}

Comprehensive health coverage is mandated by law for every citizen of Israel. The setting of this study was a busy primary care clinic which is part of one of the country's four

*Address correspondence to this author at the Department of Internal Medicine "C", Tel Aviv Sourasky Medical Center, 6 Weizman Street, Tel Aviv 64239, Israel; Tel: +972-3-6973766; Fax: +972-3-6973929;

E-mail: drcharach@012.net.il major health maintenance organizations. The average number of patients seen on an ordinary day by a family health care physician in an outpatient clinic is around 50 (5-7 per hour).

The study was conducted from January 1995 through December 2005. All patients with symptoms raising the suspicion of CHD were enrolled in to the study (according to inclusion and exclusion criteria) and scheduled for further evaluation that is outlined below to verify the existence of CHD. The study was approved by the local ethics committee.

\section{INCLUSION CRITERIA}

All individuals with symptoms raising the suspicion of CHD were eligible. These symptoms were: chest pain (retrosternal, substernal including a feeling of pressure in the chest, squeezing, heaviness, burning, aching, knife like), dyspnea, neck pain, jaw pain, back pain, palpitation, sweating, nausea, vomiting, syncope, shoulder pain, arm pain and cough, or a combination thereof.

\section{EXCLUSION CRITERIA}

Excluded from the study were patients who were younger than 40 years of age because of a low risk for CHD, as were those with confirmed trauma to the chest or neck, hyper- or hypothyroidism, previous coronary event, psychiatric disease, permanent pacemaker, malignancy, acute and severe renal failure, hepatic disease, severe pulmonary disease (e.g., chronic obstructive pulmonary disease [COPD], established active peptic disease or acute infectious disease. Finally, patients with duration of complaints of less than 5 minutes [6] were excluded because of low probability of CHD. 
Table 1. Patients' General Data

\begin{tabular}{|c|c|c|c|}
\hline & $\begin{array}{l}\text { Group 1: CHD Confirmed } \\
\qquad(n=216)\end{array}$ & Group 2: CHD Ruled Out $(n=234)$ & p value \\
\hline Age, years & $69.7 \pm 11.2($ range: $40-91)$ & $65.2 \pm 12.1($ range $40-90)$ & $<0.0001$ \\
\hline Gender: male $\%$ & 54.4 & 53.8 & NS \\
\hline HTN \% & 40.0 & 18.8 & $<0.0001$ \\
\hline Smokers $\%$ & 29.8 & 35.0 & NS \\
\hline BMI & $25.1 \pm 3.9$ & $24.5 \pm 3.3$ & NS \\
\hline $\mathrm{TC} \mathrm{mg} / \mathrm{dL}$ & $220.8 \pm 54.0$ & $196.1 \pm 34.8$ & $<0.0001$ \\
\hline $\mathrm{HDL} \mathrm{mg} / \mathrm{dL}$ & $43.0 \pm 8.8$ & $43.5 \pm 9.7$ & NS \\
\hline
\end{tabular}

CHD, coronary heart disease; DM, diabetes mellitus; HTN, hypertension; BMI, body mass index; TC, total cholesterol, HDL-c, high-density lipoprotein-cholesterol; LDL-c, low-density lipoprotein cholesterol; Tg, triglycerides

For each patient a complete medical history was taken according to the Rose questionnaire for the purpose of standardizing the anamnesis [7]. Participants with previous visits to this clinic had their records reviewed in order to retrieve data regarding previous_history of CHD and cardiac risk factors, such as diabetes mellitus, hypertension [HTN], a body mass index $(\mathrm{BMI})>25$, the lipid profile (total cholesterol [TC], high density lipoprotein-cholesterol [HDL-c], lowdensity lipoprotein cholesterol [LDL-c], triglycerides $[\mathrm{Tg}]$ ), and descriptive features of a coronary event, all of which were recorded. The complete physical examination included measurement of blood pressure, height and weight, chest auscultation, and calculation of the BMI. Based on our findings, the patients were scheduled for one or all of the following: ECG (if this had not been done during the initial visit to the primary care facility), stress tests, nuclear perfusion imaging (thallium scan), or coronary angiography or they were sent to a hospital emergency room (ER) for ECG and blood creatinine phosphokinase (CPK)/troponin determination. Some patients with imminent or established acute myocardial infarction underwent coronary angiography during hospitalization, without stress test or a thallium scan.

A follow-up visit is routinely scheduled 1-3 months after the first visit following the establishment or exclusion of CHD by the previously mentioned methods. Most of the patients were first sent to noninvasive modalities because of the probability of extracardiac symptoms. To avoid false negative findings (thallium scan or ergometry), we decided to use the 3-year follow-up findings to determine which of the patients initially diagnosed as not having CHD later developed CHD: their data were excluded from analysis.

\section{STATISTICAL ANALYSIS}

The association between demographic parameters and clinical symptoms was examined using the Student's t-test, Chi-Square test, Fisher's exact test and Pearson Correlation Coefficient according to the measurement scale of the variables. Multivariate logistic regression models were applied to the data in order to study the relationship between each risk factor: demographic (age, gender), clinical (non-insulindependent diabetes mellitus [NIDDM], HTN, smoking, BMI), lipid profile (TC, HDL, LDL, Tg) and symptoms (jaw pain, back pain, palpitations, vomiting, syncope). The level of association is presented as Odds Ratio (OR) and 95\% Confidence Interval (CI). Statistical significance was set at $\mathrm{p}<0.05$. The SPSS for Windows software, Version 12.0 was used for the analysis.

\section{RESULTS}

A total of 558 consecutive outpatients with CHD-related symptoms were eligible for participation in this prospective study according to the exclusion and inclusion criteria. Of them, 109 were excluded for technical reasons, noncompliance, or lack of follow-up information and those with negative thallium scan or treadmill ergometry who developed ischemic CHD during the ensuing 3 years ( 2 patients). The remaining 449 patients who presented symptoms associated with CHD during the 10-year study period were divided in two groups: Group 1 consisted of 216 patients (117 [54.4\%] males and 98 females [45.6\%]) with angiographically established CHD and Group 2 consisted of 233 patients (126 males [53.8\%] and 108 females [46.2\%] in whom CHD was ruled out. Eighty six patients in Group 1 were diagnosed as having acute coronary syndrome (ACS), 32 had acute myocardial infarction and 98 had stable angina pectoris. The characteristics of the two groups are presented in Table $\mathbf{1}$. The mean age for Group 1 was $69.7 \pm 11.2$ years (range 4091) and 65.2 \pm 12.2 years (range 41-90) for Group 2 $(\mathrm{p}<0.0001)$. Eighty-six $(40 \%)$ Group 1 patients had confirmed HTN, a figure which was 2.1 times the number of HTN patients in Group $2(18.8 \%$; $\mathrm{p}<0.0001)$. Patients in group 1 had $33 \%$ and $13 \%$ higher total cholesterol and LDLcholesterol, respectively $(\mathrm{p}<0.0001)$.

Fig. (1) summarizes the overall patterns of symptoms reported by patients in both study groups. As expected, the leading symptom in both groups was chest pain (172 [80\%] 


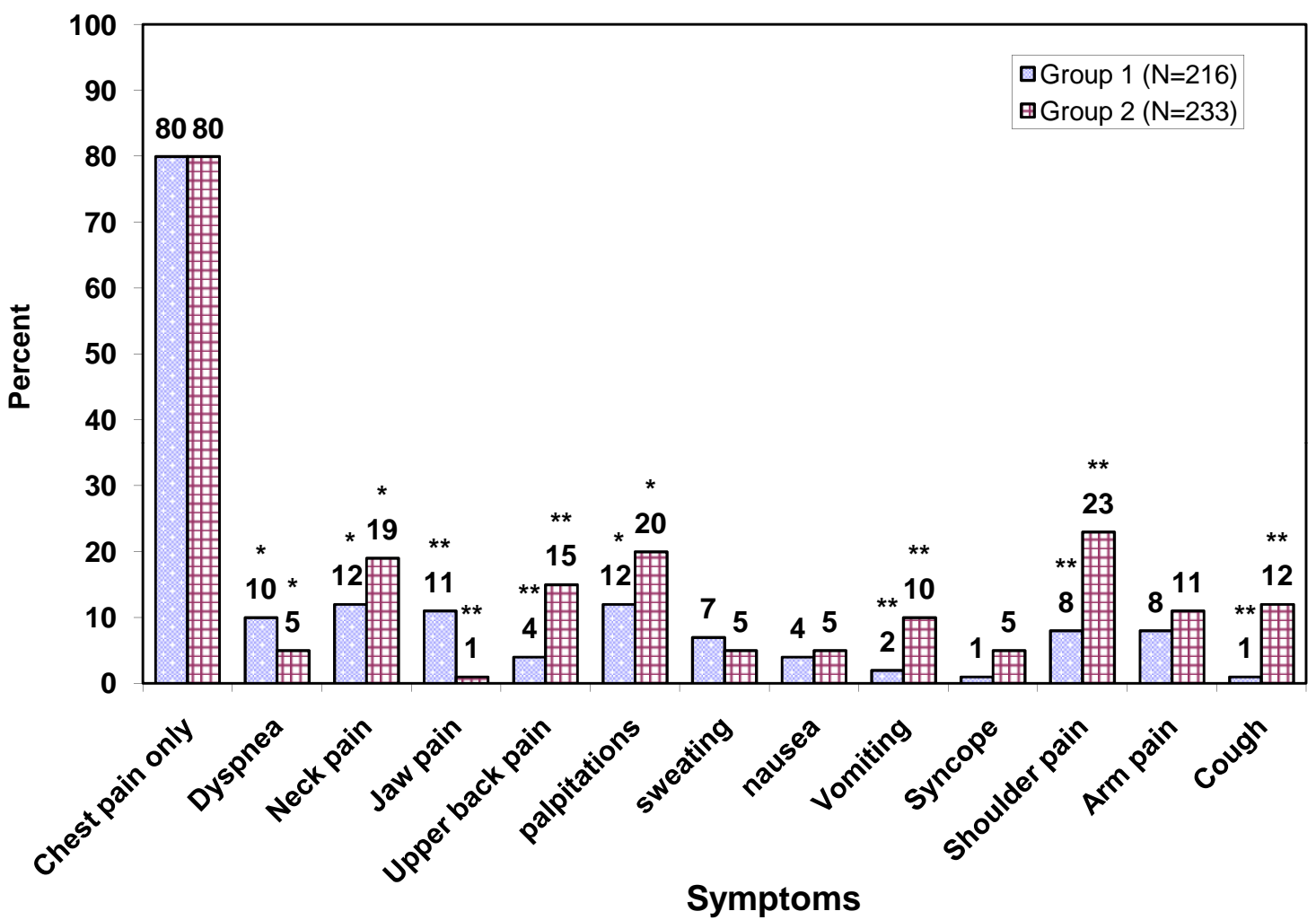

Fig. (1). The overall patterns of symptoms reported by patients in Group 1 (patients diagnosed as having coronary heart disease [CHD]) and Group 2 (patients not diagnosed as having CHD). *-p<0.005, ** $\mathrm{p}<0.0001$.

in Group 1 and 188 [80.3\%] in Group 2, (NS). The respective Group 1 and 2 differences reached a level of significance for other symptoms: dyspnea (21 [10\%] vs 11 [5\%]), $\mathrm{p}=0.037)$ and jaw pain $(23[11 \%]$ vs $3[1 \%], \mathrm{p}<0.0001)$. Unexpectedly, palpitations were recorded for 25 (12\%) Group 1 patients and for $46(20 \%)$ Group 2 patients $(\mathrm{p}<0.001)$. Patients $\leq 65$ years had more commonly jaw pain than older patients $8 \%$ vs $4 \%(\mathrm{p}<0001)$.

In order to establish the sensitivity of the symptoms for more precise diagnosis, we examined them in combination. Fig. (2) shows the differences between various symptom combinations presented by the patients in both study groups. The only significant differences between Groups 1 and 2 emerged from the symptom combinations which were more frequent in the CHD group: chest pain+dyspnea $=18(8 \%)$ vs $8(3 \%)$, respectively, $(\mathrm{p}=0.025)$, chest pain + jaw pain $=15$ $(7 \%)$ vs $1(1 \%)(\mathrm{p}<0.0001)$ and dyspnea+sweating $=6(3 \%)$ vs $0(0 \%)(\mathrm{p}=0.015)$. Unexpectedly the following combinations were significantly less frequent in group 1: chest and shoulder+arm pain $=15(7 \%)$ vs $52(22 \%) \quad(\mathrm{p}=0.01)$, chest+back pain $=9(4 \%)$ vs $30(13 \%)(\mathrm{p}=0.001)$, chest pain+cough $=2(1 \%)$ vs $24(10 \%)(\mathrm{p}<0.0001)$ and chest pain+palpitations $=11(5 \%)$ vs $27(12 \%)(\mathrm{p}=0.015)$.

Multivariate logistic regression analyses were carried out to examine if the differences in symptom presentation were either attenuated or eliminated altogether after adjustment for age, CHD-related symptoms and risk factors (Table 2). The most prominent finding was that patients with jaw pain had
11.7 times more risk to have CHD than patients without jaw pain when other symptoms and risk factors were equal. $\mathrm{Pa}-$ tients with HTN had a 2.9 risk for CHD compared to patients without HTN. The regression analysis of symptom combinations and risk factors confirmed that patients with CHD had chest pain with concomitant jaw pain 21 times more than patients without CHD (Table 3). Other variables, such as age, HTN, BMI >25, LDL-c >160 mg/dL, HDL-c <43 $\mathrm{mg} / \mathrm{dL}$, and hypertriglyceridemia $>150$ also had an increased association with CHD in the symptoms combination regression analysis. The OR's of the other symptoms were low. Sensitivity and specificity in percents of the some single symptoms were: dyspnea $10 \%$ and $90 \%$, neck pain $14 \%$ and $81 \%$, jaw pain $11 \%$ and $90 \%$, palpitation $9 \%$ and $91 \%$, respectively, in comparison to chest pain $80 \%$ and $20 \%$. Sensitivity and specificity of the several combined symptoms were:chest pain+dyspnea $8 \%$ and $97 \%$, chest pain + shoulder pain $24 \%$ and $78 \%$, chest pain + jaw pain $7 \%$ and $99 \%$ respectively.

Other symptoms (single and combined) were not tested because of small number of patients.

There were no significant differences in the presenting symptoms of patients with ACS, myocardial infarction or stable angina pectoris.

A total of 17 patients in Group 1 died (cardiac-related death), 6 in hospital and 11 during the 6 months following hospitalization. Ten patients died in Group 2 during follow 


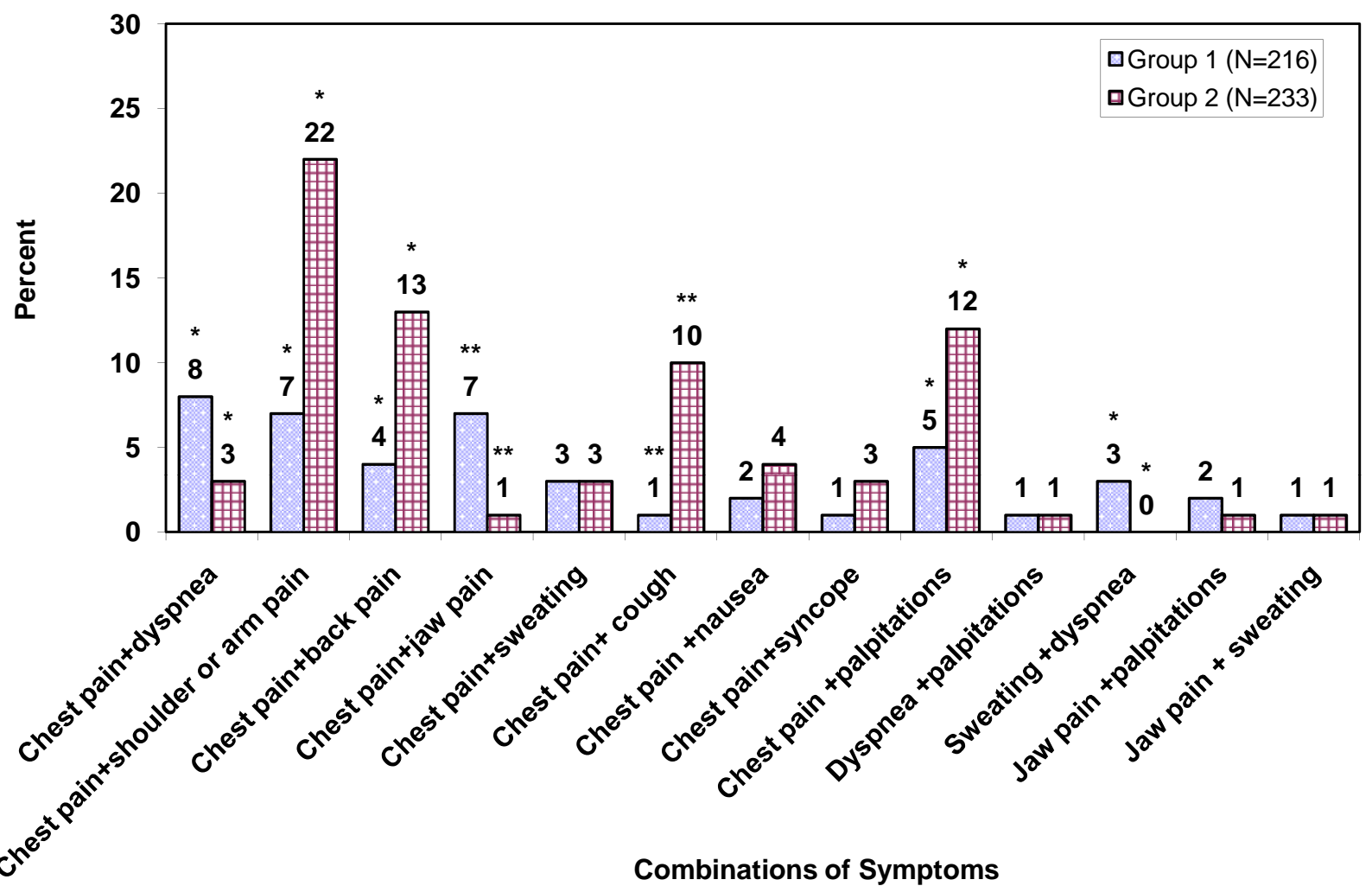

Fig. (2). The prevalence of the various symptom combinations in Group 1 (patients diagnosed as having coronary heart disease [CHD]) and Group 2 (patients not diagnosed as having CHD).*-p<0.005, ** $\mathrm{p}<0.0001$.

Table 2. Adjusted Odds Ratio of symptoms and risk factors for Coronary Heart Disease (CHD).

\begin{tabular}{|c|c|c|c|}
\hline Symptoms and Risk Factors of CHD & Odds Ratio & 95\% C.I. (Lower) & $\begin{array}{c}\text { 95\% C.I } \\
\text { (upper) }\end{array}$ \\
\hline Age & 1.059 & 1.032 & 1.086 \\
\hline Jaw pain & 11.725 & 2.347 & 58.561 \\
\hline Back pain & 0.208 & 0.073 & 0.592 \\
\hline Vomiting & 0.273 & 0.084 & 0.894 \\
\hline Syncope & 0.161 & 0.023 & 1.130 \\
\hline Shoulder pain & 0.355 & 0.169 & 0.746 \\
\hline Cough & 0.114 & 0.027 & 0.475 \\
\hline Low-density lipoprotein & 1.079 & 1.058 & 1.101 \\
\hline High-density lipoprotein & 0.917 & 0.884 & 0.950 \\
\hline Triglycerides & 1.006 & 1.001 & 1.011 \\
\hline
\end{tabular}

C.I., confidence interval

up, but only 2 were cardiac related deaths. Three hundred and sixteen patients (including all patients in group1) underwent coronary angiography, 332 (165 in group1) underwent thallium scans, and 271 (108 in group1) underwent ergome- try. Thirty two patients in group 1 underwent urgent coronary angiography due to ECG changes. Eighteen patients had angiography due to ECG changes + troponin/CPK elevation. 
Table 3. Adjusted Odds Ratio of Symptoms Combination and Risk Factors for Coronary Heart Disease (CHD)

\begin{tabular}{|c|c|c|c|}
\hline Symptoms and Risk Factors & Odds Ratio & $\begin{array}{c}\text { 95\% C.I. } \\
\text { (Lower) }\end{array}$ & $\begin{array}{c}\text { 95\% C.I. } \\
\text { (Upper) }\end{array}$ \\
\hline \hline Age & 1.057 & 1.032 & 1.082 \\
\hline Combined chest+jaw pain & 20.772 & 178.676 & 4.415 \\
\hline Hypertension & 2.582 & 0.642 & 0.829 \\
\hline Body mass index & 0.730 & 1.064 & 1.104 \\
\hline Low-density lipoprotein & 1.084 & 0.919 & 0.950 \\
\hline High-density lipoprotein & 0.919 & 1.006 & 1.011 \\
\hline Triglycerides & 1.006 & \\
\hline
\end{tabular}

\section{DISCUSSION}

The present study evaluated typical and atypical symptoms of CHD and their combinations in order to streamline and enhance the correct the diagnosis of a life-threatening disorder that can often be vague and mimic other conditions. This is an important but difficult topic to research. Most previous studies on the role of symptoms in diagnosing CHD have been based upon emergency room presentations (1-3) or disease registries, and therefore subject to referral bias. That this study chose consecutive patients in a primary care setting makes it unique. The strength of the study resides in the large number of patients enrolled, in-depth longitudinal analysis of a number of non-specific symptoms and long follow up period. The study intended to improve the ability of family physicians to identify symptoms reflecting myocardial ischemia. We are unaware of a previous such investigation in the primary care setting.

Significant differences between patients with proven CHD and those found not to have CHD were demonstrated for several presenting symptoms and their combinations. These differences emphasize the need to provide clearcut information for primary physicians and clinical cardiologists with better tools to facilitate early diagnosis and appropriate triage for further testing or urgent hospitalization. We took special note of the importance of atypical symptoms: in addition to classical symptoms, [3] which are often nonspecific. The current work also emphasizes the importance of less common yet more specific symptoms for CHD, such as jaw pain $11 \%$ and dyspnea $10 \%$, and combinations of symptoms, such as chest pain + jaw pain $7 \%$ and dyspnea + sweating $3 \%$. These emerged as being more predictive for CHD, especially in elderly patients. The question to what extent the more specific symptoms might be affected by environmental and genetic factors is still not clear, however, we don't think that ethnicity is related to this, because our patients cohort was ethnically homogenic.

The indicated symptoms appear to represent more specific means for diagnosing CHD, however a significant number of patients presented less specific symptoms especially: palpitations $-12 \%$, neck pain $-12 \%$, shoulder pain $8 \%$, arm pain- $8 \%$, and such combined symptoms as chest pain+dyspnea- $8 \%$, chest pain +shoulder or arm pain- $7 \%$ and chest pain+palpitations-5\%. These symptoms were not significantly different between patients with confirmed CHD and those found not to have CHD. In contrast, the group of "non cardiac" patients (Group 2) unexpectedly showed an increased frequency of palpitations, neck pain, back pain, and shoulder pain, and the combinations of chest + shoulder pain, chest + back pain, and chest pain + palpitations. Also unexpected was the lack of differences between the two groups in the established risk factors for CHD i.e. smoking and NIDDM. (This can be explained by the relatively small number of "smokers" and NIDDM patients in the study group). We found that part of atypical symptoms in our patients showed exceedingly high specificity, yet low sensitivity e.g. dyspnea, palpitation, jaw pain, shoulder pain.

In line with the findings of Meshak, et al. [3] who reported sex difference in presenting symptoms, we found that male patients had jaw pain and sweating more commonly than females. Women were more likely to describe experiencing fatigue, dyspnea, dizziness, upper back pain, palpitations and cough. In addition patients $\leq 65$ years more frequently described jaw pain, palpitations + jaw pain and chest pain + jaw pain thus highlighting a common knowledge that older people experience fewer and more subtle symptoms $[1$, 2]. No significant differences were found in the presenting symptoms between patients with ACS, acute myocardial infarction or stable angina pectoris. Thus the same symptoms can clearly be common to all three syndromes and, in real time, it is very difficult to distinguish between them without ECG and appropriate laboratory work-up.

Most of our patients (>80\%) experienced the "classical" symptoms of chest pain.

When we looked at atypical symptoms, such as jaw pain alone or in combination, we found a prevalence of $11 \%$. This is higher than the 9\% reported by Sampson and Cheitlin's study on differential diagnosis of cardiac pain [8]. Jaw pain was, additionally, highly predictive of CHD in our study group $(\mathrm{p}<0.0001)$.

Jaw pain of cardiac origin needs to be differentiated from other kinds of mandibular pain. our patients complained of pain in the lower part of jaw, mostly in the mandibular angle on the left side $[9,10]$. The pain was not sharp as dental pain, and did not radiate to other parts of the head and neck like 
the ear or the pharynx [9]. It was also characteristically periodic and frequently induced by physical exertion, strong emotion or eating.

In the Corpus Christi Heart Project [3], that compared symptoms of acute myocardial infarction between Mexican Americans and non-Hispanic whites found jaw+arm symptoms in $58 \%$ of the former and $65 \%$ in the latter. Our impression is that the incidence of jaw pain is underestimated by clinicians and this underlines the importance of taking a careful patient history [11].

Efforts aimed at reducing prehospital delay in referral of patients with CHD to the ER might be more efficient with greater attention paid to "uncommon" and underestimated symptoms of CHD. Obviously careful assessment of chest pain, ECG and subsequent lab-tests must be royal road to streamlining diagnosis of CHD, however, since lab-tests are not available in primary community clinic physicians are faced with the challenge of identifying patients with high probability of CHD based on clinical presentation only. The presence of jaw pain, dyspnea or a combination of these in addition to positive history for hypertension and dyslipidemia have a higher specificity than classical chest pain for the presence of CHD. Identifying a host of possible cardiac symptoms and appropriate rapid response to them are essential for the improving of CHD outcome [11,12].

\section{LIMITATIONS}

Limitations of this studies are the inability to pinpoint an accurate clinical clue (s) for identifying coronary events according to the symptom/sign presenting to the primary physician in a setting where ECG and laboratory tests are usually not available. In other words, we did not succeed in defining clinical criteria that indicate with high precision the need for urgent referral to acute care facility. We were unable to substantiate the diagnosis of CHD on angiography in all cases for the sake of standardization because we had to rely on the insurer approval of such a procedure which was not invariably given in the field. It was not ethical to send patients to evaluation tests without indications whatsoever.

To avoid false negative findings in the group 2, we used the minimum 3-year follow-up findings to determine which of the patients initially diagnosed as not having CHD later developed CHD: their data were excluded from analysis.

Identifying a host of cardiac symptoms and appropriate prompt response are essential for the improving of $\mathrm{CHD}$ outcome [11,12].

\section{IMPLICATIONS}

Our findings are intended to guide physicians in primary care clinics in enhancing earlier diagnosis of CHD. We advise paying special attention to atypical symptoms and combinations of symptoms which we list specifically. We also urge greater dissemination of information to the public, explaining that heart attacks manifest themselves by diverse symptoms and not by chest pain alone. Recognizing other possible cardiac symptoms and taking appropriate and rapid measures are essential for improving CHD outcome [12, 13].

\section{ACKNOWLEDGMENT}

Esther Eshkol is thanked for editorial assistance.

\section{REFERENCES}

[1] Goldberg R, Goff D, Cooper L, et al. Age and sex differences in presentation of symptoms among patients with acute coronary disease: the REACT trial. Coron Artery Dis 2000; 11: 399-407.

[2] Goldberg RJ, O'Donnell C, Yarzebski J, Bigelow C, Savageau J, Gore JM. Sex differences in symptom presentation associated with acute myocardial infarction: a population-based perspective. Am Heart J 1998; 136: 189-95.

[3] Meshak A, Goff D, Ramsey D, Linares A, Reyna R, Pandey P. Comparison of reported acute myocardial infarction in Mexican Americans versus non-hispanic whites (the corpus christi heart project). Am J Cardiol 1998; 82: 1329-32.

[4] Verani M, Mahmarian J, Hixson J, Boyce T, Staudacher R. Diagnosis of coronary artery disease by controlled coronary vasodilation with adenosine and thallium 201 scintigraphy in patients unable to exercise. Circulation 1990; 82: 80-7.

[5] Kannel W, Feinleib M. Natural history of angina pectoris in the framingham study. Am J Cardiol 1972; 29: 154-63.

[6] McCurren R, Mikhail M, Ensign L, Frederiksen S. Does time of onset and duration of chest pain reliably exclude the diagnosis of acute myocardial infarction in chest pain observation patients? Acad Emerg Med 2001; 5: 540-3.

[7] Rose GA. The diagnosis of ischemic heart pain and intermittent claudication in field surveys. Bull World Health Organ 1962; 27: 645-58.

[8] Sampson J, Cheitlin M. Pathophysiology and differential diagnosis of cardiac pain. Prog Cardiovasc Dis 1971; 13: 507-18.

[9] Sandler NA, Ziccardi V, Ochs M. Differential diagnosis of jaw pain in the elderly. J Am Dent Assoc 1995; 126: 1263-72.

[10] Drinnan A. Differential diagnosis of orofacial pain. Dent Clin North Am 1978; 22: 73-87.

[11] LaCroix A, Haynes S, Savage D, Havlik RJ. Rose questionnaire: angina among united states black, white and mexican-american women and men. Am J Epidemiol 1989; 129: 669-85.

[12] Jonson JA, King K. Influence of expectations about symptoms delay in seeking treatment during myocardial infarction. Am J Crit Care 1995; 4: 29-35.

[13] Burnett R, Blumenthal J, Mark D, Leimberger J, Califf R. Distinguishing between early and late responders to symptoms of acute myocardial infarction. Am J Cardiol 1995; 75: 1019-22.

(C) Charach et al.; Licensee Bentham Open.

This is an open access article licensed under the terms of the Creative Commons Attribution Non-Commercial License (http://creativecommons.org/licenses/by-nc/3.0/) which permits unrestricted, non-commercial use, distribution and reproduction in any medium, provided the work is properly cited. 